53 PREDICTING EPIDURAL SPACE SPREAD USING ULTRASOUND COLOR DOPPLER IMAGING IN INTERLAMINAR EPIDURAL STEROID INJECTION: A PROSPECTIVE OBSERVATIONAL STUDY

YJ Kim*, H Kim, H-J Kim, WU Koh. Asan Medical Center, Seoul, Korea, Republic of

10.1136/rapm-2021-ESRA.53

Background and Aims While the use of fluoroscopy-guided transforaminal epidural steroid injection (TFESI) to help spread the injectate toward the ventral side has increased, this procedure entails a radiation risk. Recently, ultrasound has been widely applied in the medical field; among ultrasound methods, color Doppler is useful for predicting the direction of the injectate. This study describes a novel technique employing color Doppler to help predict epidural space spread in interlaminar epidural steroid injection (ILESI).

Methods This observational study prospectively enrolled 35 patients scheduled for lumbar epidural steroid injection (ESI). Ultrasound-guided epidural injection was conducted and realtime images using color Doppler were recorded during injections of $5 \mathrm{~mL}$ of $0.1 \%$ ropivacaine containing contrast dye with dexamethasone. Fluoroscopy-guided TFESI was performed if it was difficult to perform the procedure based on ultrasound images.

Results The analysis included 30 images from 30 patients. The observed sensitivity, specificity, positive predictive value, and negative predictive values of the ultrasound color Doppler were $100 \%, 89.5 \%, 84.6 \%$, and $100 \%$, respectively. The agreement with ultrasound color Doppler was 93.3\%.

Conclusions The main advantage of ultrasound-guided ILESI is the lack of radiation exposure and contrast medium

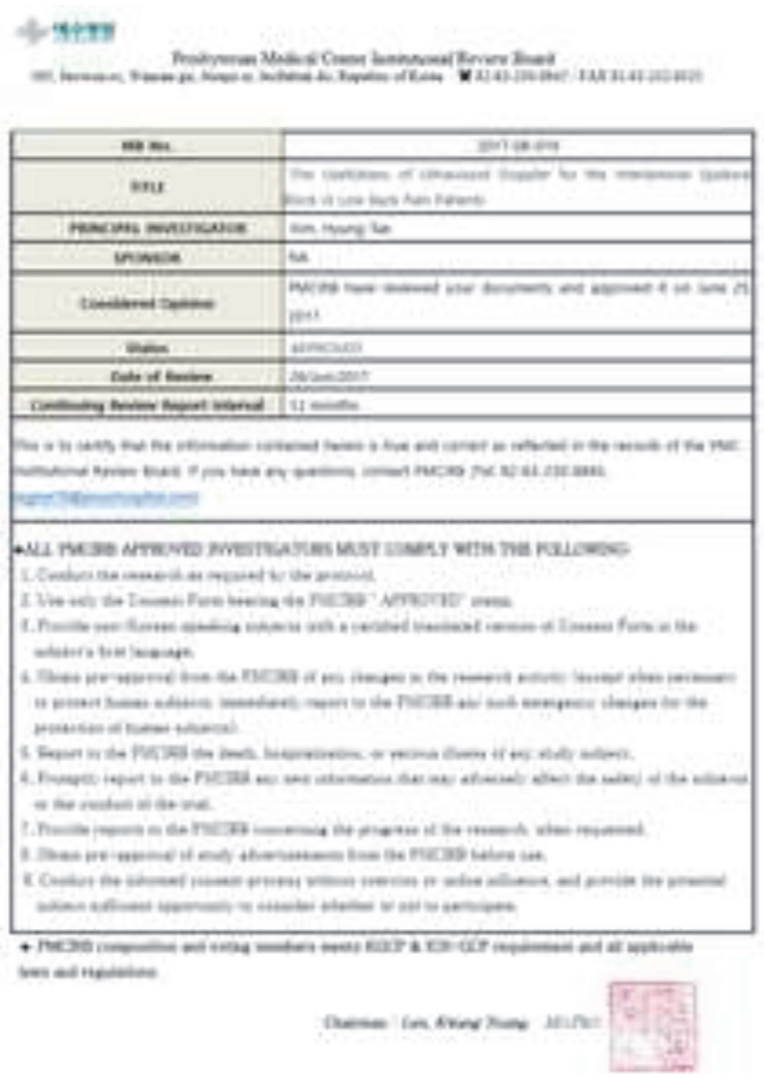

requirement. Color Doppler may be a reliable imaging modality to predict epidural space spread during ultrasound-guided ILESI. It is worth predicting the spread in the anterior epidural space (AES) by first attempting ultrasound-guided ESI. If the injectate has not spread to the AES, fluoroscopy-guided TFESI may be a good option after confirming the improvement of the patient's symptoms.

\section{LOW-DOSE, OPIOID-FREE SUBARACHNOID BLOCK AND US-GUIDED SUPRAINGUINAL FICB AS AN ANAESTHETIC/ ANALGESIC TECHNIQUE IN PATIENTS WITH ASYMPTOMATIC, MILD TO MODERATE AORTIC STENOSIS UNDERGOING HIP FRACTURE SURGERY}

H Abrahams*, R Matos-Puig. GJGM Regional Hospital, Stanger, South Africa

\subsection{6/rapm-2021-ESRA.54}

Background and Aims Aortic Stenosis (AS) is a relatively common valvular lesion in patients over 75.The significant risks associated with surgery may be mitigated by choice of anaesthetic technique and careful perioperative planning. Haemodynamic goals broadly aim to avoid hypotension and tachycardia and to preserve coronary perfusion pressure and systemic vascular resistance. Neuraxial blockade is traditionally taught to be a (relative) contraindication in this particular group of patients.

Aim: To assess perioperative pain scores and intraoperative hypotension after a technique comprising a low-dose, slowtitrated SAB and US-guided SIFI FICB in patients with mildmoderate disease as evidenced on TTE undergoing hip fracture surgery.

Methods IRB was obtained.

8 patients were enrolled prospectively over 6 months (Nov 2019 - April 2020)

All patients had a preoperative TTE to categorise disease severity.

Invasive arterial monitoring was instituted preoperatively in all patients.

US-guided SIFI FICB followed by a SAB (25G pencil point needle) was performed.

$(30 \mathrm{ml} \mathrm{0.25 \%}$ isobaric Bupivicaine and $7.5 \mathrm{mg}$ hyperbaric Bupivicaine x $1 \mathrm{~min}$, respectively).

No opioids were used.

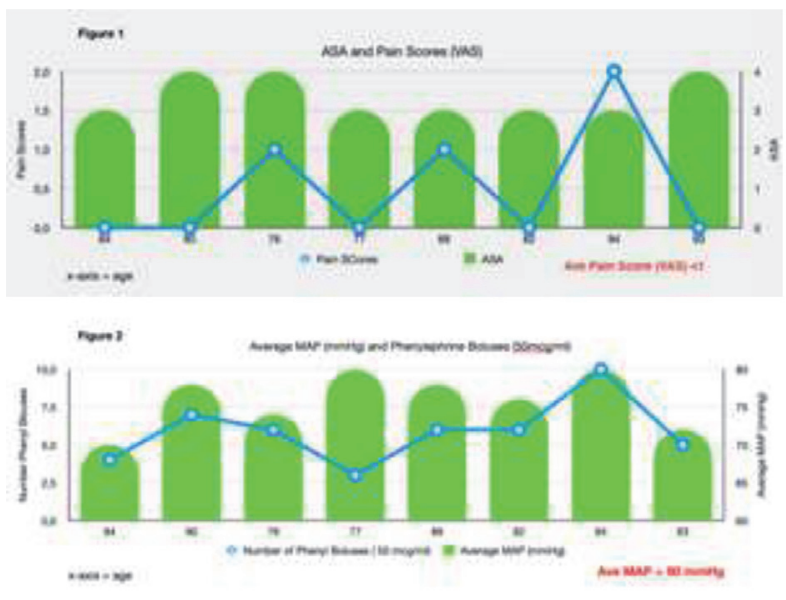

Abstract 54 Figure 1 